\title{
A new device to noninvasively estimate the intraocular pressure produced during ocular compression
}

This article was published in the following Dove Press journal:

Clinical Ophthalmology

12 January 2016

Number of times this article has been viewed

\author{
Michael S Korenfeld ${ }^{1,2}$ \\ David K Dueker ${ }^{3}$ \\ 'Comprehensive Eye Care, Ltd., \\ ${ }^{2}$ Department of Ophthalmology \\ and Visual Sciences, Washington \\ University, Washington, MO, USA; \\ ${ }^{3}$ Hamad Medical Corporation, Doha, \\ Qatar
}

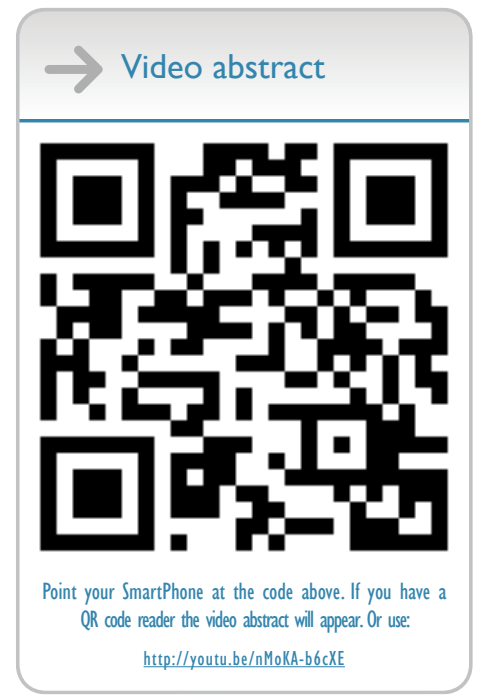

Correspondence: Michael S Korenfeld Comprehensive Eye Care, Ltd, 90I East 3rd Street, Washington, MO 63090, USA Tel +l 6363903999

Fax +l 6363903959

Email michaelkorenfeld@hotmail.com
Purpose: To describe a noninvasive instrument that estimates intraocular pressure during episodes of external globe compression and to demonstrate the accuracy and reliability of this device by comparing it to the intraocular pressures simultaneously and manometrically measured in cannulated eyes.

Methods: A thin fluid-filled bladder was constructed from flexible and inelastic plastic sheeting and was connected to a pressure transducer with high pressure tubing. The output of the pressure transducer was sent to an amplifier and recorded. This device was validated by measuring induced pressure in the fluid-filled bladder while digital pressure was applied to one surface, and the other surface was placed directly against a human cadaver eye or in vivo pig eye. The human cadaver and in vivo pig eyes were each cannulated to provide a manometric intraocular pressure control.

Results: The measurements obtained with the newly described device were within $\sim 5 \%$ of simultaneously measured manometric intraocular pressures in both a human cadaver and in vivo pig eye model for a pressure range of $\sim 15-100 \mathrm{mmHg}$.

Conclusion: This novel noninvasive device is useful for estimating the intraocular pressure transients induced during any form of external globe compression; this is a clinical setting where no other devices can be used to estimate intraocular pressure.

Keywords: glaucoma, intraocular pressure, tonometer, ocular compression

\section{Introduction}

Many noninvasive devices that estimate intraocular pressure have been invented over the last century. Most deform the globe and correlate the force responsible for the deformation to the pressure within the eye. Most physically contact the globe while in use (Schiotz, Goldmann, Draeger, Perkins, Mackay-Marg, Tono-pen, pneumatonometer, and Maklakov) while others do not (air puff tonometer). The mechanisms and scientific premises that underlie these instruments are well-described. ${ }^{1}$ Another germane device is the contact lens tonometer. ${ }^{2}$ This transparent device is applied directly to the globe while in use. In order for the device to produce meaningful measurements, it must be held in a specific orientation upon the eye by an examiner who must also ensure that the space between the contact lens and the cornea is filled with fluid. If the lens is not well centered on the cornea, the three internal pressure sensors will measure very different externally applied appositional forces. External appositional forces can be indirectly applied to the globe by applying force to the contact lens and the resulting artificially raised intraocular pressure can be estimated; ocular pulsations are also measureable with this device and are seen superimposed on the continuous intraocular pressure tracings. Because the device is transparent, it can be used to observe 
the retinal vasculature while the appositional force is increased. It is therefore suited to perform ophthalmodynomometry.

Unfortunately, it would be impossible to properly utilize any of these devices while a subject's eyelids were closed and their globe was being externally compressed. Since this clinical situation does arise, a novel ocular pressure estimator (OPE) was invented with this application in mind. Generally, it is accepted that the aforementioned noninvasive devices only "estimate" the intraocular pressure. The gold standard for measuring "true" intraocular pressure is the cannulated eye, which provides direct manometric measurements. The OPE was therefore tested in the most rigorous fashion, on cannulated eyes that were simultaneously providing manometric measurements.

To better acquaint the reader with the hypothesis that underlies the mechanism of the OPE, the following examples and schematic diagrams are offered.

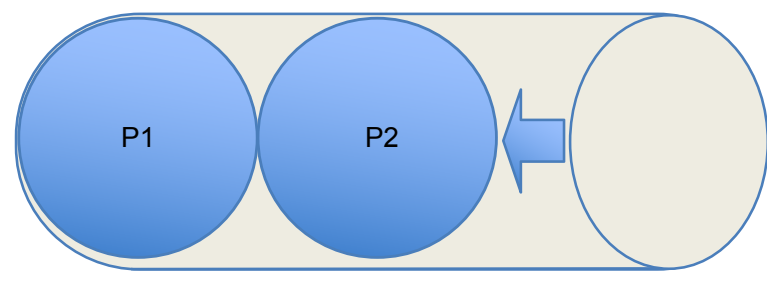

Example 1: Two tennis balls in contact, with an external force acting on P2: P1 = P2.

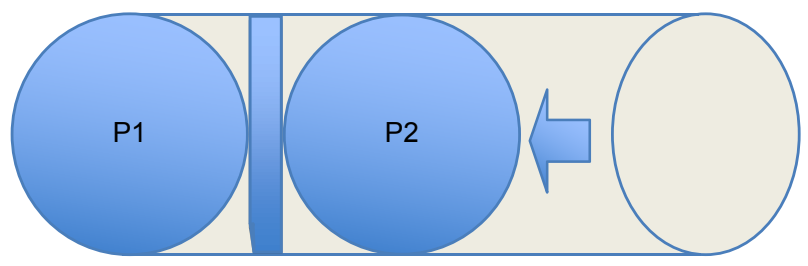

Example 2: Two tennis balls in contact, separated by a noncompressible septum, with an external force acting on P2: $\mathrm{P} 1=\mathrm{P} 2$ = pressure within the septum.

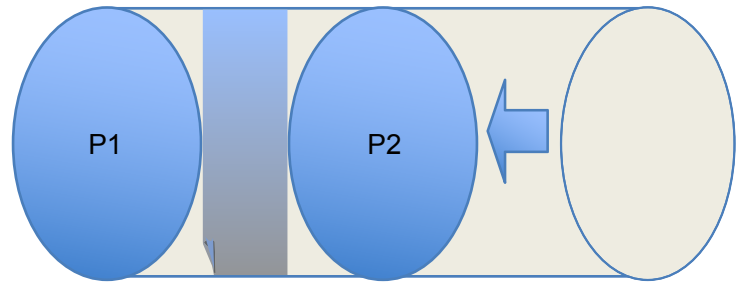

Example 3: Two tennis balls in contact, separated by a compressible septum, with an external force acting on P2. The septum thins until maximal compression has occurred, and then the system is analogous to Example 2, and the septum behaves in a noncompressible manner.

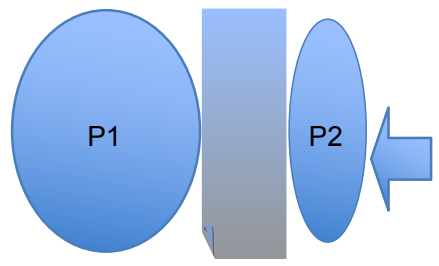

Example 4: $\mathrm{P} 1$ is a human globe in vivo. The compressible septum is the eyelid. P2 is the fluid-filled bladder of the OPE. In use, as the outer wall of the fluid-filled bladder is compressed by an external force, then the compressible aspect of the eyelid becomes maximal, and then the eyelid behaves as a noncompressible septum (like Example 2), and the pressures in the globe = compressed eyelid pressure = OPE pressure.

Figure I A schematic that describes the hypothesis of the OPE is shown.

Notes: Example I shows the general principle of the hypothesis. Example 2 adds a noncompressible septum between two vessels with similar wall compliances. As long as the septum is noncompressible and all three elements are in physical contact, the thickness of the septum does not alter the equalization of the pressures inside both vessels when external pressure is directly applied to just one of the vessels. Example 3 posits a compressible septum interposed between these same two vessels. As soon as the external pressure applied to the outer wall of one vessel produces maximal compression in the septum, the system becomes analogous to Example 2 . Example 4 assigns the theoretical elements described in Example 3 to the actual elements found in the experimental design.

Abbreviation: OPE, ocular pressure estimator. 
than the diameter of the tennis balls, and the outermost ball is compressed, the force of the compression will initially only be partially conveyed to the second ball, until all of the potential compression within the septum has occurred, and then the septum acts like it is noncompressible (like in Example 2), and then the pressure inside both balls, and the collapsed septum, becomes the same (Figure 1).

Example 4: The OPE's fluid-filled bladder was constructed from flexible, but inelastic plastic sheeting. This was chosen to match the wall compliance, but not the thickness, of an intact globe. It is impractical to construct a pressure transducer from a cadaver globe and the volume of such a cadaveric transducer anterior to a person's intact globe would prevent meaningful measurements in clinical practice. The fluid-filled bladder of the OPE was designed to address these issues. Using the fluid-filled bladder of the OPE in vivo in humans over their closed eyelids is analogous to Example 3 above. Once the eyelid under the fluid-filled bladder was fully compressed by the external force applied to the outer wall of the fluid-filled bladder, the pressure of the globe, the fully compressed lid, and the OPE became the same. The lid thickness is not important, only that it is fully compressed before the pressures in all three locations become the same (Figure 1).

\section{Methods}

The fluid-filled bladder of the OPE (Figure 2) was constructed by heat sealing a flexible but inelastic plastic sheet around a polyethylene tube (PE-50, Intramedic, Clay Adams, Parsippany, NJ, USA) so that all heat-sealed junctions were water tight up to pressures of $200 \mathrm{mmHg}$. The portion of the polyethylene tube that was fused into the plastic bladder was friction fit onto a short segment of a blunt-ended shaft of a broken 23-gauge needle, which served to bridge the fluid-filled bladder portion to the length of the polyethylene tubing that was attached to the three-way stopcock, $0.5 \mathrm{~m}$ away. The stopcock was attached by a high-pressure hose to a pressure transducer (Model PX-105, Omega Engineering, Inc., Stamford, CT, USA). A bottle of sterile balanced salt solution (BSS, Alcon Surgical, Inc., Fort Worth, TX, USA) was connected to the three-way stopcock with high pressure hosing. A continuous fluid column without air bubbles was thus formed between the calibrated BSS bottle, the stopcock, the pressure transducer, the polyethylene tubing, and the fluid-filled plastic bladder (Figure 3). The amplifier circuitry was designed to produce a linear amplification of the pressure transducer's signal output. The signal output produced by the pressure transducer was sent to this amplifier circuit and then to a strip chart recorder (Omega Engineering,

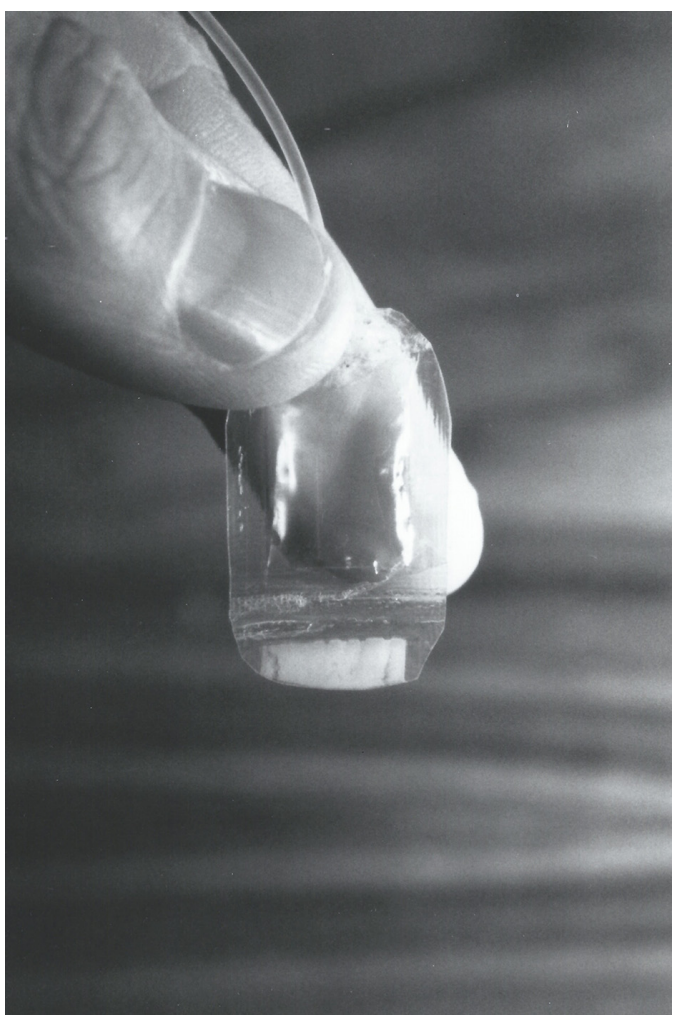

Figure 2 The portion of the OPE that contacts the eye is shown in the fluid-filled and ready-to-use state.

Note: The mechanical properties of the plastic used for the construction of this bladder were chosen to match the compliance properties of the eye wall: flexible, but relatively inelastic.

Abbreviation: OPE, ocular pressure estimator.

Inc., RD-MV/Volt module with a series RD-2000 Function Recorders Mainframe).

In order to validate the OPE, an additional control setup was also constructed. It consisted of all of the same components, and the output of the two amplifier circuits was electronically matched. The only difference between the two setups was that the OPE ended distally with the heat-sealed plastic bladder and the control device ended distally with a needle for intraocular manometric readings. The needle for the human cadaver eye had the fluid column emerge from the distal sharp end, while the fluid column for the anterior segment needle ended at a slot drilled half way along the needle's shaft so that the aqueous humor completed the fluid continuum; the sharp end of the needle was occluded with cyanoacrylate glue for the anterior segment experiments prior to placement.

The plastic bladder was held at the same level as the pressure transducer during calibrations. The calibrating BSS bottle was raised above the zero level (transducer level) in height increments corresponding to $10 \mathrm{mmHg}$. A linear response was confirmed over the range of 0-120 mmHg. 


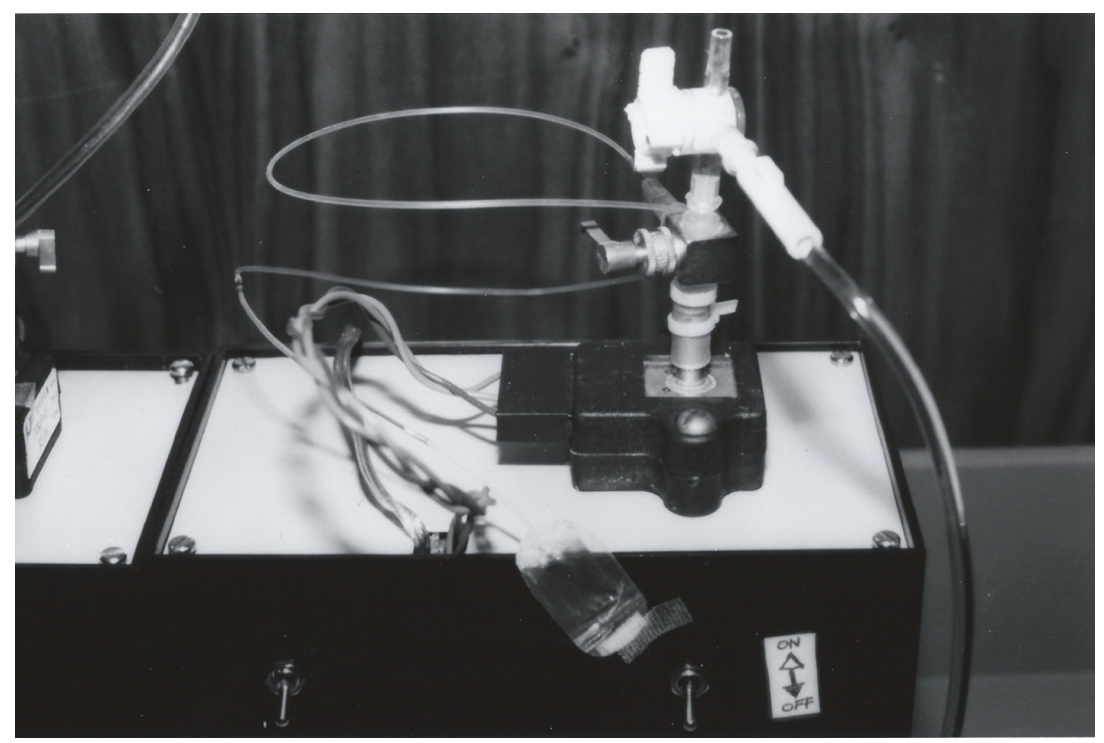

Figure 3 The OPE apparatus is shown.

Notes: The apparatus consisted of a bottle of fluid for calibration (not seen), a three-position stopcock, a pressure transducer (the black structure directly below the stopcock, bolted to the white surface), a linear amplifier circuit (the black box with the white top), a strip chart recorder, and a measurement line which was connected to the stopcock with polyethylene tubing. Two identical setups were constructed, except the experimental OPE line ended distally with a fluid-filled bladder (shown here) and the control line ended distally with a needle for intraocular cannulation.

Abbreviation: OPE, ocular pressure estimator.

After calibration of the transducer, the system was brought to zero pressure by lowering the BSS bottle, and the BSS bottle was then cut off from the system with the stopcock. This left the fluid-filled bladder in sole connection with the transducer at nominal zero pressure. At this point, the fluid-filled bladder was $\sim 1.5 \mathrm{~mm}$ thick and was ready for use (Figure 2 ). The following experiments were performed to validate the OPE.

For the first experiment, a 19-gauge needle was broken from the hub and was fitted into polyethylene tubing (PE-100, Intramedic, Clay Adams) that was connected to the control transducer/amplifier setup previously described. A fresh human donor globe was cannulated through the length of the optic nerve with this needle. The fluid in the needle lumen formed a liquid continuum with the vitreous body. The needle was passed while fluid was running through it to reduce the chance of tissue plugging the lumen and to eliminate air bubbles in the line. Once in place, the calibrating bottle was elevated from the level of the globe in $10 \mathrm{mmHg}$ increments to $200 \mathrm{mmHg}$ to confirm the lack of leaks and to confirm the expected linear increments of intraocular pressure with known increments in bottle height. The calibrating bottle was lowered to the equivalent of $15 \mathrm{mmHg}$ and the stopcock system was used to isolate the globe and the transducer from the calibrating bottle. The globe was then placed within a mock orbit that consisted of a rigid Styrofoam box filled with a hydrated sponge with holes carved out for the globe and the optic nerve. A latex surgical glove was draped over the globe to simulate the closed eyelid (Figure 4).
The OPE was prepared and calibrated as previously described. The fluid-filled bladder was placed on the mock eyelid and digital pressure was applied through it to the globe. Variable amounts of digital pressure were applied in a manner similar to the technique used after glaucoma filtration surgery to enhance filtration function. Pressures within the plastic bladder and the manometrically cannulated globe were simultaneously and continuously recorded (Figure 5).

In another experiment, the OPE was evaluated on the eye of a live, anesthetized pig. A $40 \mathrm{~kg}$ pig undergoing terminal anesthesia for a urologic procedure was used for this experiment. Ethical and legal approval was obtained prior to the commencement of the study. This was provided by the University of Missouri-Columbia, Columbia, Missouri. All experiments performed in support of this paper were performed in accordance with the tenets of the Declaration of Helsinki and followed relevant institutional and national guidelines and regulations. Animal use in this study conformed to the Association for Research in Vision and Ophthalmology Resolution on the Use of Animals in Research.

The manometric control measurements for this experiment were obtained with the same setup that was used for the control arm of the human cadaver eye experiments, except that in this experiment, manometric readings were taken from the anterior chamber through a 23-gauge side-slotted needle.

Prior to cannulating the anterior chamber, the nictitating membrane was surgically excised, and a $8 \mathrm{~mm}$ lateral canthotomy was performed to improve access to the cornea. 


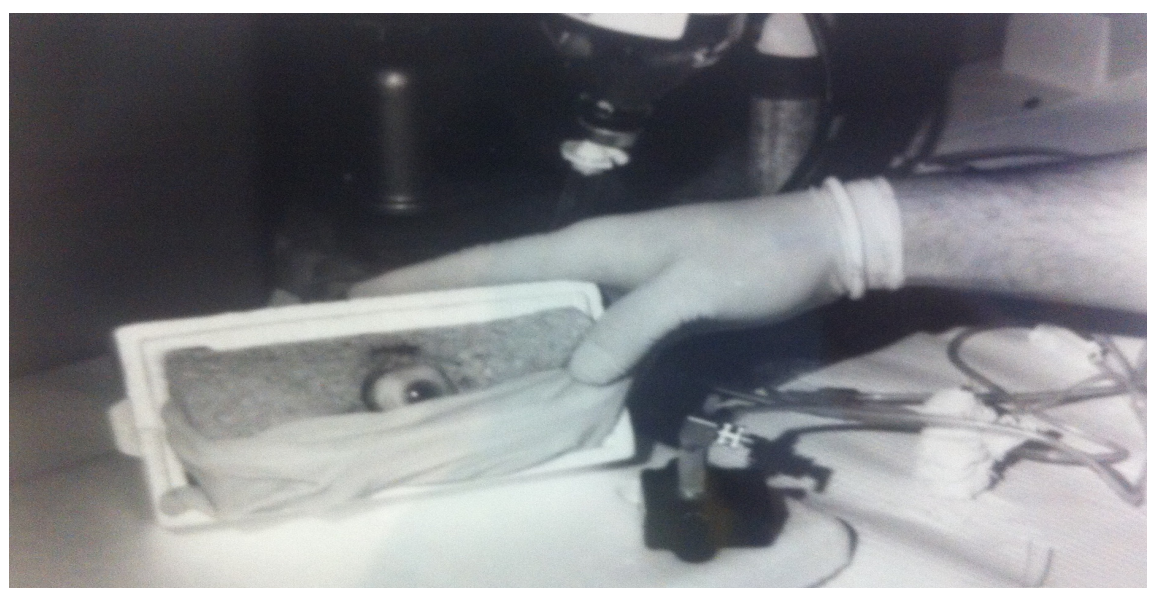

Figure $4 \mathrm{~A}$ fresh human cadaver eye was mounted within a mock orbit that consisted of a Styrofoam box filled with a moist sponge.

Notes: Manometric intraocular pressure readings were made after the vitreous cavity was cannulated. The plastic bladder is shown centered over the cornea, interposed between the experimenter's fingertips and a mock eyelid. The tracings produced during this experiment are shown in Figure 5.

The anterior chamber was cannulated by passing the sideslotted needle through the temporal cornea, across the anterior chamber, and out through the nasal cornea. The needle was passed while fluid was running through it to reduce the chance of tissue plugging the lumen and to eliminate air bubbles in the line. The slot drilled in the needle was oriented downward so it would be least likely to encounter tissue during external compressions. The needle was connected via tubing to the control transducer/amplifier setup previously described. The calibrating BSS bottle was raised and lowered in increments equal to $10 \mathrm{mmHg}$ to test for any leaks and to confirm a linear transducer response. Following this, the BSS bottle was brought to a height equivalent to $15 \mathrm{mmHg}$, and the calibrating BSS bottle was isolated from the transducer with the stopcock, and opened to connect the transducer to the line leading to the needle that ended distally in the anterior chamber of the pig eye. The eye pressure was then recorded without external perturbation to confirm stability.

The fluid-filled bladder was then applied to the closed eyelid centered over the cornea. Digital pressure was applied to the exposed surface of the plastic bladder over a wide range of pressures. Simultaneous and continuous tracings

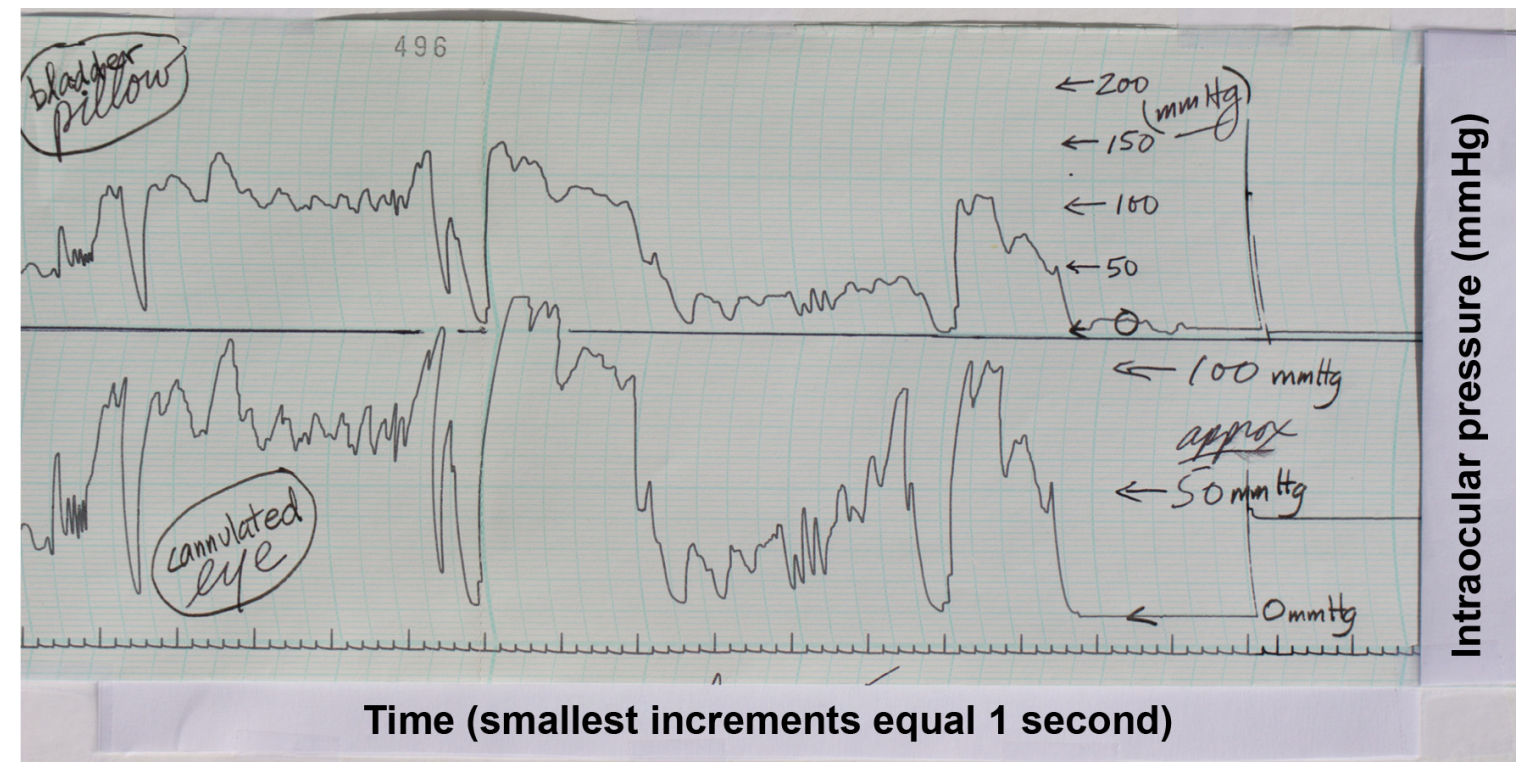

Figure 5 The simultaneous tracings produced during the human cadaver eye experiments are shown.

Notes: The upper pressure tracing recorded the OPE output and the lower control tracing recorded the manometrically measured intraocular pressure from the cannulated eye. The vertical scales for the pressure readings in the two setups are in $\mathrm{mmHg}$, but are not identical. The pressure scale of the lower tracing is more expanded than the upper scale, and as a result, the morphology of the two tracings is not identical. There was approximately a 2-second delay between the two setups and short-term pressure fluctuations were simultaneously seen in both systems. When the time delay between the two setups and the different pressure scales are considered, the pressures measured in the control and experimental setups were typically within $5 \%$ of each other for pressures between 15 and $100 \mathrm{mmHg}$.

Abbreviation: OPE, ocular pressure estimator. 


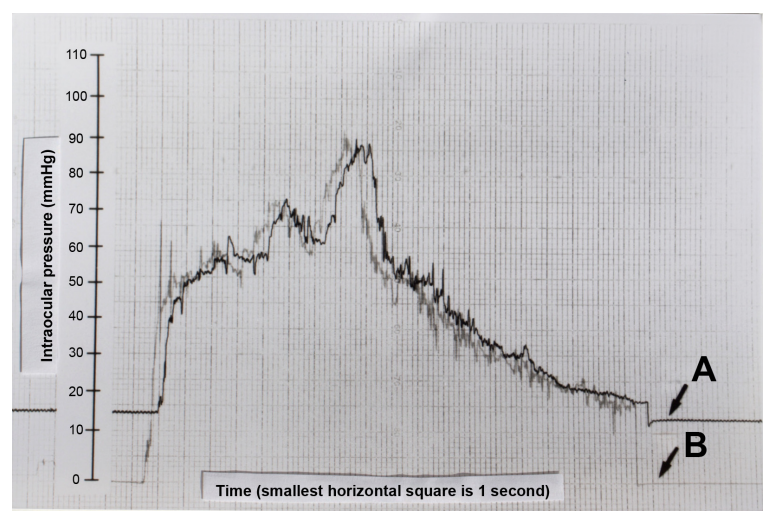

Figure 6 The tracings produced during the in vivo intracameral pig eye experiment are shown.

Notes: The scales to the left of both tracings represent the reference pressure increments obtained during calibration and are in $\mathrm{mmHg}$. In this experiment, the pressure scales are the same for the experimental and control setups. The tracing of the cannulated eye is labeled $\mathbf{A}$, and the tracing generated by the OPE is labeled $\mathbf{B}$. There is approximately a 2-second delay between the two setups. There are $\sim 20$ seconds of manometric intraocular pressure shown before and after compressions in tracing A. Considering the time delay between the two setups, the pressures measured in the control and experimental setups were typically within $5 \%$ of each other for pressures between 17 and $90 \mathrm{mmHg}$.

Abbreviation: OPE, ocular pressure estimator.

of the OPE and cannulated pig eye were made for induced elevations of pressure above the $20 \mathrm{mmHg}$ baseline. After the experiment, the pressure within the OPE returned to baseline and the pressure within the pig eye came to a new steady state below its initial baseline (Figure 6).

Attempts were also made to obtain simultaneous estimates of intraocular pressure in live human subjects using the OPE and either a hand-held applanation tonometer (Perkins MKII Applanation Tonometer, Clement Park, Inc., Columbus, OH, USA) or a Tono-pen (BioRad, Oculab, Inc., Glendale, CA, USA). Unfortunately, there was not sufficient access to or exposure of the cornea to allow for this method of calibration, and thus, no data are presented.

\section{Results}

The manometrically measured intraocular pressures in the human cadaver eye were typically within the $5 \%$ of the pressures estimated by the OPE in the range of $15-100 \mathrm{mmHg}$ (Figure 5). Even though the human cadaver eye was calibrated to pressures as high as $200 \mathrm{mmHg}$, calibration pressures greater than $100 \mathrm{mmHg}$ were not recorded due to the limited scale of the control channel on the strip chart recorder. It is therefore difficult to know if the OPE output remained quantitatively matched to control pressures over $100 \mathrm{mmHg}$, even though the two tracings closely followed each other over $100 \mathrm{mmHg}$. There was approximately a 2 -second time delay between the two setups.
For the in vivo pig eye experiment, the "baseline" intraocular pressure was "locked into" the globe with the stopcock at $\sim 15 \mathrm{mmHg}$ (tracing A). The pressure within the fluid-filled bladder, when held at the level of the corneal apex but without eye contact, was $0 \mathrm{mmHg}$ (tracing B) (Figure 6). When the fluid-filled bladder was placed upon the eye, and pressure was exerted across the globe, the pressure within the bladder rose until it reached the "locked in" pressure of the globe. As the pressure of the external compression rose above the "locked in" pressure, the tracings of the OPE and the cannulated eye were typically within $5 \%$ of each other for a range of $\sim 17-90 \mathrm{mmHg}$. Once the OPE was in contact with the globe, and the external pressure applied to the fluid-filled bladder was equal to or greater than the intraocular pressure, the experimental system became analogous to Example 4 in the schematic of Figure 1. When the fluid-filled bladder was removed from the globe, the pressure within it returned $0 \mathrm{mmHg}$ and the cannulated eye returned to an intraocular pressure plateau that was $2-3 \mathrm{mmHg}$ below the precompression "locked in" pressure. The lower postcompression plateau was felt to result from a tonographic effect, which was expected in this experiment with a live animal. Note the stability of the manometrically measured intraocular pressure prior to and after external ocular compression, as well as the fine superimposed respiratory variations.

\section{Discussion}

It was the intention of this investigation to construct and validate an instrument that could be used to provide some quantification of the intraocular pressure transients that are produced during external ocular compression. The OPE was the result of this effort.

Data obtained from the cannulated eye experiments provide the best substantiation for the validity of the OPE. The experiment with a human cadaver eye had the advantage of placing the manometric needle at a site distant from the corneal compression, minimizing the chance that the two pressure sensing instruments would disturb each other while in use. The OPE was also validated in a live animal model. This experiment confirmed the close agreement of the manometrically measured intraocular pressure and the output of the OPE, first demonstrated with the human cadaver eye, in a mock orbit. When the time delay between the two setups and the different pressure scales are considered, the pressures measured in the control and experimental setups were very closely matched.

The cannulated eye preparations were intact throughout all experimentation. No leaks of the plastic bladder or sites of 
globe cannulation were ever visualized despite induced pressures of up to $200 \mathrm{mmHg}$. The soundness of the preparation was confirmed when the postcompression control pressure in the human eye returned to the same level that was "locked in" by the BSS bottle and stopcock before external compressions were performed. There was no tonographic effect expected in a human cadaver eye, and none was seen.

In the pig eye, a tonographic effect was observed. The postcompression pressure plateau was several $\mathrm{mmHg}$ lower than the precompression plateau. In addition to the tonographic effect, the fine respiratory pressure variations were also observed to return. The postcompression pressure tracing not only confirms the lack of leaks, but further suggests that the lumen of the intracameral needle was not plugged with tissue. The pressure scale in the pig eye experiment provided a better opportunity to observe these more subtle intraocular pressure effects.

This novel device should be more useful than previously described devices for estimating intraocular pressures during periods of time when the eye experiences externally applied forces. Clinically relevant periods of time when this occurs include during sleep, eye rubbing, and during the digital ocular massage maneuver used by glaucoma surgeons and their patients after trabeculectomy to improve bleb performance. In the future, it is contemplated that a more "user-friendly" device analogous to the OPE would be constructed using the same principles as the ones validated here, but without stopcocks and tubing. This modification will make using the OPE more simple and approachable for clinicians and patients, for use in the clinic and at home.

\section{Disclosure}

The authors report no conflicts of interest in this work.

\section{References}

1. Ritch R, et al. Tonometers and tonometry. In: The Glaucomas. St. Louis: The C.V. Mosby Company; 1989:303-312.

2. Dekker PW, Robert YC, Kanngiesser H, Pirani P, Entenmann B. Principles of contact lens tonometry. Int Ophthalmol. 1999;22(2):105-111.
Clinical Ophthalmology

\section{Publish your work in this journal}

Clinical Ophthalmology is an international, peer-reviewed journal covering all subspecialties within ophthalmology. Key topics include: Optometry; Visual science; Pharmacology and drug therapy in eye diseases; Basic Sciences; Primary and Secondary eye care; Patien Safety and Quality of Care Improvements. This journal is indexed on

Submit your manuscript here: http://www.dovepress.com/clinical-ophthalmology-journal

\section{Dovepress}

PubMed Central and CAS, and is the official journal of The Society of Clinical Ophthalmology (SCO). The manuscript management system is completely online and includes a very quick and fair peer-review system, which is all easy to use. Visit http://www.dovepress.com/ testimonials.php to read real quotes from published authors. 